\title{
Comparative effect of propofol versus fentanyl in controlling acute renal colic and its hydronephrosis in patients admitted to the hospital
}

\author{
Saeed Hayatia ${ }^{\text {|Reza Yazdania }}$ |Mohsen Esmaeilib $^{\text {|Shabnam Kazemic }}$ |Seyed Ashkan Tabibzadeh \\ Dezfulia,*
}

${ }^{a}$ Assistant Professor, Trauma and Emergency Medicine Research Center, Hormozgan

University of Medical Sciences, Bandar Abbas, Iran

${ }^{b} M D$, Hormozgan University of Medical Sciences, Bandar Abbas, Iran

cEmergency Medicine Specialist, Hormozgan University of Medical Sciences, Bandar Abbas, Iran

\begin{abstract}
Background and purpose: Renal colic (RC) is observed in the urinary system and known with sudden and serious pain. Various analgesics are usually used for alleviating pain in the emergency department. This study compared the effect of Propofol with Fentanyl in controlling acute RC of patients admitted to the hospital. Methods: In this study, 150 patients referred to Shahid Mohammadi Hospital (Bandar Abbas-Iran) for the treatment of RC were selected. The basal pain values of the patients were investigated by the Visual Analogue Scale (VAS) that were in range from 0 up to 10 at the 0 th, (immediately before drug administration), $15^{\text {th }}$, and $30^{\text {th }}$ minutes. The zero value shows lack of pain and highest value (10) means the severe pain. Results: The results showed that VAS value was significantly higher in VAS0 compared to VAS10 and VAS30 for all the groups $(P<0.05)$. The results also showed a significant difference between Morphine, Propofol and Fentanyl for VAS10 and VAS30 $(P<0.05)$. The results showed that values for VAS were 3.66, 1.19 and 5.05 for Morphine, Fentanyl and Propofol groups, respectively. The values for VAS30 were 2.85, 1.19 and 3.52 for Morphine, Fentanyl and Propofol groups, respectively. The lowest value was observed for Fentanyl and the highest value was observed in Propofol group for VAS10 and VAS30. Conclusion: The best response was observed in Fentanyl group and it can be recommended using Fentanyl for controlling acute renal colic and its hydronephrosis in patients admitted to the hospital.
\end{abstract}

\section{KEYWORDS}

Analgesics; hydronephrosis; renal colic; visual analogue scale.

\section{Introduction}

Renal colic (RC) usually occurs under conditions such as kidney stones and is known with sudden and serious pains [1]. It is an emergency condition that patients report it as a painful condition [2]. It is known with some signs, such as abdominal and back pain and hematuria [2]. Decreasing pain is first steps for the treatment of acute renal colic [3].
Nonsteroid anti-inflammatory drugs (NSAIDs) and opioids are commonly used as analgesics for alleivating pain. Hydronephrosis is not a true disease, but it is commonly used for identifying the amount of renal dilation from another disease or status that may be present and alsocan involve the both kidneys [1]. The agents have significant analgesic effects and can help to relief pain in the early period [4]. Analgesics are intravenously (iv) and 
intramuscularly (im) administrated in the emergency department [4]. Propofol (2,6diisopropylphenol) is a sedative agent that is usually used in emergency medicine. However, it has some disadvantages such as loss of airway reflexes, hypoventilation, apnea, and hypoxia [5]. It is a highly lipid-soluble anesthetic that has antioxidant activity against lipid peroxidation under in vitro and in vivo conditions [6]. It inhibits renal renal injury through down-regulating inducible nitric oxide synthase expression [7] and protects HK-2 human kidney proximal tubular epithelial cells against stress induced by $\mathrm{H}_{2} \mathrm{O}_{2}$ [8]. Other synthetic opioid analgesic is Fentanyl that is an opioid agonist. It is an ideal drug, because it has high solubility in fat and can be administrated by traditional parenteral route [4]. It can be administrated via im, iv (bolus injection, infusion, patient-controlled analgesia), neuroaxial (epidural, intrathecal), transdermal, transmucosal (oral or intranasal) and inhalation routes [9]. It is used as an effective analgesic under emergency conditions $[10,11]$ and has not any side effects such as nausea, dizziness and altered consciousness [11]. It has advantages such as providing a very high level of analgesia and also shows its effect immediately after administration [2]. So far, any study has not been conducted to compare the effects of Propofol Versus Fentanyl on RC. This study, for first time, compared the effect of Propofol and Fentanyl in controlling acute RC of patients admitted to the hospital.

\section{Materials and methods}

\section{Patients}

All the procedures used in this study were approved by Ethics committee approval (IR_HUMS_REC_1398.051) in Faculty of Medicine, Hormozgan University of Medical Sciences which was planned as a randomized double blind study. In this study, 150 patients referred to the the Shahid Mohammadi Hospital (Bandar Abbas-Iran) for the treatment of RC were selected. The inclusion criteria were as follows; 1 ) Consent of patients for participation in the study, 2) Patients with an age range of 18-70 years, 3) Patients with medium and/or severe pain (Score of $>5$ ), 4) Non-responding to the treatment of anti-pain in the Emergency and 5) confirmation of RC by urinary analysis, KUB image, sonography and CT scan. Exclusion criteria included: 1) Patients that did not complete consent for participation in this study, 2) lack of diagnosis by imaging and laboratory tests, 3) Patients with history of cardiovascular, blood pressure, liver, paroxysm Pregnant women, nursing mothers, and consumption of psychotherapy drugs, 4) Allergy to egg and 5) using sedative drugs $6 \mathrm{~h}$ before of bedridden. Demographic information including age, sex, weight and renal hydronephrosis were registered. Before administration of drug, the basal pain values of the patients were investigated by the Visual Analogue Scale (VAS) in values from 0 up to 10 at the 0th, (immediately before drug administration), $15^{\text {th }}$, and $30^{\text {th }}$ minutes. Value of zero means lack of pain and 10 means the most pain.

\section{Drug selection}

The drugs used in the this study were numbered on the basis of alphabetical order, A, B, C,... . The drugs were allocated to one of the study groups on the basis the alphabetical words. The drugs were prepared by someone outside the emergency department. Only this person knewwords allocated to drugs. Administration of drugs were conducted by a nurse in the hospital. The nurse administrated the drug through iv, infusion and slow routes during 5 minutes. The Propofol and Fentanyl were prepared from Braun Melsungen and Darou Pakhsh, respectively. The lowest administration levels for drugs were as follows; Fentanyl (2 $\mu \mathrm{g} / \mathrm{kg}$ body weight), Propofol $(0.30 \mu \mathrm{g} / \mathrm{kg}$ body weight $)$ and morphine $(0.10 \mathrm{mg} / \mathrm{kg}$ body weight $)$. Following administration of drugs, the VAS 
was assessed in 10 and 30 minutes after administration.

\section{Statistics}

The data were analyzed by the Chi-square test, T-independent and ANOVA procedure. To calculate demographic characteristics, Chisquare test was used. Pair-comparisons was performed by T-independent. Comparing between 3 groups was performed by one-way ANOVA procedure. We used SPSS 22.0 software for analysis of data. $\mathrm{P}<0.05$ was considered as significant.

\section{Results and discussion}

\section{Demographic characteristics}

The results for demographic characteristics of the patients studied are shown in Table 1 . The results showed that 34 patients (68\%) and 16 patients (32\%) were man and woman in morphine group, 29 patients (58\%) and 21 patients (42\%) were man and woman in Fentanyl group and 28 patients (56\%) and 22 patients (44\%) were man and woman in Propofol group, respectively. In addition, the means for age and weight were 33.61 years and $73.16 \mathrm{~kg}$. The results also showed that 21 patients (42\%), 27 patients (54\%) and 24 patients (48\%) had ureteral stones in Morphine, Fentanyl and Propofol groups, respectively. The results also showed that 3 patients (6\%), 4 patients (8\%) and 2 patients (4\%) had hydronephrosis history in Morphine, Fentanyl and Propofol groups, respectively. In addition, 3 patients (6\%), 7 patients (14\%) and 3 patients (6\%) had diabetes in Morphine, Fentanyl and Propofol groups, respectively.

TABLE 1 Demographic characteristics of patinets studied

\begin{tabular}{cccc}
\hline & Morphine & Fentanyl & Propofol \\
\hline Sexuality (Man) & $34(68 \%)$ & $29(58 \%)$ & $28(56 \%)$ \\
Sexuality (Woman) & $16(32 \%)$ & $21(42 \%)$ & $22(44 \%)$ \\
Ureteral stones & $21(42 \%)$ & $27(54 \%)$ & $24(48 \%)$ \\
Hydronephrosis history & $3(6 \%)$ & $4(8 \%)$ & $2(4 \%)$ \\
HTN & $5(10 \%)$ & $6(12 \%)$ & $10(20 \%)$ \\
Diabetes & $3(6 \%)$ & $7(14 \%)$ & $3(6 \%)$ \\
Cardiovascular & $0(0 \%)$ & $0(0 \%)$ & $1(2 \%)$ \\
\hline
\end{tabular}

\section{The VAS value}

The data for comparison of VAS values at the 0 th 15 th and 30th minutes in patients with acute hydronephrosis are shown in Table 2. The results showed that for all the groups, VAS value was significantly higher in VAS0 compared to VAS10 and VAS30 $(P<0.05)$. The results showed, as VAS increases from 0 to 30 , the mean decreases $(\mathrm{P}<0.05)$. There was no significant difference between groups for VAS0 $(P>0.05)$. The results showed that mean for VAS0 was 8.80, 9.04, 9.23 and 9.01 for
Morphine, Fentanyl and Propofol groups and total, respectively. There was significant difference between Morphine, Propofol and Fentanyl for VAS10 and VAS30 $(P<0.05)$. The results showed that values for VAS were 3.66, 1.19 and 5.05 for Morphine, Fentanyl and Propofol groups, respectively. The values for VAS30 was 2.85, 1.19 and 3.52 for Morphine, Fentanyl and Propofol groups, respectively. The lowest value was observed for Fentanyl and the highest value was observed in Propofol group for VAS10 and VAS30. 
TABLE 2 Comparison of VAS values at the 0th 15th and 30th minutes in patients with acute hydronephrosis

\begin{tabular}{ccccc}
\hline Group & VAS & Mean & \multicolumn{2}{c}{ P-values } \\
\hline \multirow{4}{*}{ Morphine } & 0 & 8.80 & Vs Fentanyl $=0.514$ & Vs Propofol $=0.157$ \\
& 10 & 3.66 & Vs Fentanyl $=0.000$ & Vs Propofol $=0.000$ \\
& 30 & 2.85 & Vs Fentanyl $=0.000$ & Vs Propofol $=0.002$ \\
Fentanyl & 0 & 9.04 & Vs Morphine $=0.514$ & Vs Propofol $=0.689$ \\
& 10 & 2.42 & Vs Morphine $=0.000$ & Vs Propofol $=0.000$ \\
& 30 & 1.19 & Vs Morphine $=0.000$ & Vs Propofol $=0.000$ \\
Propofol & 0 & 9.23 & Vs Morphine $=0.157$ & Vs Fentanyl $=0.689$ \\
& 10 & 5.05 & Vs Morphine $=0.000$ & Vs Fentanyl $=0.000$ \\
& 30 & 3.52 & Vs Morphine $=0.002$ & Vs Fentanyl $=0.000$ \\
Total & 0 & 9.01 & & 0.178 \\
& 10 & 3.62 & & 0.000 \\
& 30 & 2.69 & & 0.000 \\
\hline
\end{tabular}

\section{Discussion}

To the best of our knowledge, the present study is the first study that compares the analgesic efficacy of Fentanyl and Propofol for controlling acute $\mathrm{RC}$ and its hydronephrosis in patients with RC. On the basis of previous reports, prevalence of $\mathrm{RC}$ was 1 million patients in the United States, 7-9\% of emergency ambulatory in Eropean country [12] and totally between $12 \%$ and $15 \%$ in all over world [13]. In all the groups, the most patients' studies were male and middle age. Parallel to our findings, several studies showed that the most patients referred to the hospital were middle age and man $(14,15]$.

The results showed that morphine, Fentanyl and Propofol controlled acute RC in patients with hydronephrosis, but the best response was observed in Fentanyl group in patients with hydronephrosis. Hydronephrosis is condition for aseptic dilatation of kidney, because it obstructs outflow of urine. It is a condition that any urinary distention of the renal pelvi-calyceal system with/without obstruction to the urinary outflow distal into the renal pelvis [16]. The RC commonly occurs due to increased pressure in the upper urinary tract and/or dilatation of kidney capsule after urinary retention. With regards to previous studies, a study showed recruitment in patients with cancer that received morphine [17]. Parallel to our findings, King et al., [18] reported that morphine can alleviate pain in patients with RC.

Song et al., [8] showed propofol alleviates renal fibrosis under in vivo condition that has clinical potential in early unilateral ureteral obstruction in mice. They also showed that propofol can prevents TGF- $\beta 1$ expression and iNOS-NO production and kidney fibrosis through regulating miR-155 levels. Jalili et al., [19] in a meta-analysis supported using ketamine-propofol as an effective combination for decreasing some complications, and they suggested using ketofol as an appropriate substitution instead of propofol [19]. In the current study, the lowest efficiency was found for propofol group. It is has some limitations, such as cardiorespiratory depression [20]. The best responses were observed for Fentanyl. Some studies compared different forms of fentanyl are compared with each other or with morphine. Parallel to our findings, Deaton et al. [11] compared the efficacy of IV morphine $(0.1 \mathrm{mg} / \mathrm{kg})$ and nebulized fentanyl $(2 \mu \mathrm{g} / \mathrm{kg})$ on the basis of VAS scores at the 10th, 20th, 30 th, and 40th minutes and reported that nebulized fentanyl induced significant analgesia at all time intervals. Motamed and Maleki Verki [1] compared intravenous Lidocaine and Fentanyl in RC and showed that the mean for pain severity was not significantly different between fentanyl and 
lidocaine in the different times following administration.

\section{Conclusion}

In conclusion, this study compared efficiency of Propofol, Fentanyl and morphine in controlling acute RC and its hydronephrosis in patients admitted to hospital. Among agents used, administration of Fentanyl significantly controlled acute RC and its hydronephrosis compared to Propofol and morphine. It can be suggested using Fentanyl for controlling acute RC and its hydronephrosis in patients admitted to hospital.

\section{References}

[1] H. Motamed, M. Maleki Verki, Emerg., 2017, 5, e82.

[2] A.L. Behcet, M.M. Sunar, S. Zengin, M. Sabak, M. Bogan, B. Can, S. Kul, M.M. Oktay, S.H. Eren, Am. J. Emerg. Med., 2017, 36, 571-576.

[3] E. Phillips, S. Kieley, E.B. Johnson, M. Monga, J. Endourol., 2009, 23, 1021-1024.

[4] M. Imamoglu, A. Aygun, O. Bekar, E. Erdem, M. Cicek, O. Tatli, Y. Karaca, A. Sahin, S. Turkmen, S. Turedi, Am. J. Emerg. Med., 2017, 35, 757-763.

[5] I. Ferguson, A. Bell, G. Treston, L. New, M. Ding, A. Holdgate, Pain. Manag., 2016, 68, 574582.

[6] T.D. Runzer, D.M. Ansley, D.V. Godin, G.K. Chambers, Anesth. Analg., 2002, 94, 89-93.

[7] B. Dikmen, H. Yagmurdur, T. Akgul, M. Astarci, H. Ustun, C. Germiyanoglu, J. Anesth., 2010, 24, 73-80.

[8] L. Song, S. Shi, W. Jiang, X. Liu, Y. He, Am. J. Transl. Res., 2016, 8, 460-472.

[9] P.W. Peng, A.N. Sandler, Anesthesiol., 1999, 90, 576-599.
[10] S. Farahmand, S. Shiralizadeh, M.T. Talebian, S. Bagheri-Hariri, M. Arbab, H. Basirghafouri, Am. J. Emerg. Med., 2014, 32, 1011-1015.

[11] T. Deaton, J.D. Auten, M.A. Darracq, Am. J. Emerg. Med., 2015, 33, 791-795.

[12] S. Thakore, E.A. McGuan, W. Morrison, J. R. Soc. Med., 2002, 95, 126-129.

[13] H.H. Kim, M.K. Jo, C. Kwak, S.K. Park, Urol., 2002, 59, 517-521.

[14] M.L. Edmonds, J.W. Yan, R.J. Sedran, S.L. McLeod, K.D. Theakston, CJEM, 2010, 12, 201206.

[15] V. Chauhan, B. Eskin, J.R. Allegra, D.G. Cochrane, Am. J. Emerg. Med., 2004, 22, 560563.

[16] I. Singh, M.K. Joshi, Surgery Update, Maulana Azad Medical College, New Delhi, 2012.

[17] M. Wood, M. Ashby, A. Somogyi, B. Fleming, J. Pain. Symptom. Manage., 1998, 16, 112-120.

[18] S. King, K. Forbes, G.W. Hanks, C.J. Ferro, C.J. Chambers, Palliat. Med., 2011, 25, 525552.

[19] M. Jalili, M. Bahreini, A. Doosti-Irani, R. Masoomi, M. Arbab, H. Mirfazaelian, Am. J. Emerg. Med., 2016, 34, 558-569.

[20] J.P. Cravero, M.L. Beach, G.T. Blike, Anesth. Analg., 2009, 108, 795-804.

How to cite this article: Saeed Hayati, Reza Yazdani, Mohsen Esmaeili, Shabnam Kazemi, Seyed Ashkan Tabibzadeh Dezfuli*. Comparative effect of propofol versus fentanyl in controlling acute renal colic and its hydronephrosis in patients admitted to the hospital. Eurasian Chemical Communications, 2020, 2(5), 604-608. Link:

http://www.echemcom.com/article_101931.html 